How to build a low cost spectrometer with Tracker for teaching light spectra

This content has been downloaded from IOPscience. Please scroll down to see the full text.

2016 Phys. Educ. 51014002

(http://iopscience.iop.org/0031-9120/51/1/014002)

View the table of contents for this issue, or go to the journal homepage for more

Download details:

IP Address: 94.132.246.51

This content was downloaded on 16/08/2016 at 13:28

Please note that terms and conditions apply.

You may also be interested in:

A simple spectrophotometer using common materials and a digital camera

Eko Widiatmoko, Widayani, Maman Budiman et al.

Teaching optical phenomena with Tracker

$M$ Rodrigues and P Simeão Carvalho

Quantitative analysis of transmittance and photoluminescence using a low cost apparatus

P Onorato, M Malgieri and A De Ambrosis

Measuring the hydrogen Balmer series and Rydberg's constant with a homemade spectrophotometer

P Onorato, M Malgieri and A De Ambrosis

Demonstrations of optical spectra with a video camera

Yaakov Kraftmakher

An educational spectrograph using a digital camera as a training aid for physics students

V Kiisk

Design and evaluation of a visible-to-near-infrared electronic slitless spectrograph

Wenbo Wang and Jitendra Paliwal 


\title{
How to build a low cost spectrometer with Tracker for teaching light spectra
}

\author{
M Rodrigues ${ }^{2,5}$, M B Marques ${ }^{1,4}$ and P Simeão Carvalho ${ }^{1,2,3}$ \\ ${ }^{1}$ Departamento de Física e Astronomia, FCUP, Rua do Campo Alegre, \\ s/n, 4169-007 Porto, Portugal \\ 2 IFIMUP-IN, Rua do Campo Alegre, s/n, 4169-007 Porto, Portugal \\ ${ }^{3}$ Unidade de Ensino das Ciências, FCUP, Rua do Campo Alegre, s/n, 4169-007 Porto, \\ Portugal \\ ${ }^{4}$ INESC-TEC, Rua do Campo Alegre, s/n, 4169-007 Porto, Portugal \\ 5 Middle School of Viso, Porto, Portugal \\ E-mail: marcelojrodrigues@sapo.pt,mbmarque@fc.up.pt and psimeao@fc.up.pt
}

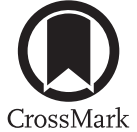

\begin{abstract}
Optics is probably one on the most exciting topics in physics. However, it also contains some of the less understood phenomena by students - the light spectra obtained from the diffraction of light. The experimental study of light spectra for studying radiating bodies, usually requests sophisticated and expensive equipment that is not normaly affordable for schools, and only a few teachers know how to measure the wavelength of light in a spectrum. In this work we present a simple and inexpensive setup, with enough accuracy for measuring light spectra to be used both in physics and chemistry classes. We show how freeware software Tracker, commonly used for teaching mechanics, can serve to measure wavelengths with about $2 \mathrm{~nm}$ of resolution. Several approaches to the calibration of different setups are also provided, depending on the degree of accuracy demanded.
\end{abstract}

\section{Introduction}

As teachers, we know how students are fascinated by optics and optical phenomena. In Middle and High school curricula, light spectra are discussed several times in different contexts such as astronomy, chemistry, optics and communications. The spectrum of a light source allows us to establish many characteristics of that source. Students use some simple spectrometers to see the different color components of white light, learn the theory of light emission and observe the emission and absorption spectra of some chemical elements. They also learn that the electromagnetic waves are classified by its frequency or wavelength, but only a few teachers know how a spectrometer really works, and how we can measure the wavelength of each color.

Nowadays it is relatively easy to produce a spectroscope and watch the light spectrum of sunlight (the rainbow colors) or of artificial light. There are lots of optical prisms and recycled materials like CDs and DVDs, or diffraction gratings in the market, at a low cost price that can disperse the white light into its components. However, spectrometers are usually expensive, and schools are reluctant to spend a significant 


\section{Rodrigues et al}

part of their budget in such equipment. There are very cheap spectroscopes in the market that allow some measurements but they have usually poor accuracy.

In this work we show how to build a low cost spectrometer with a reasonable measuring accuracy, using affordable components (e.g. commercial diffraction grating) or recycled material (DVDs), a digital camera and the free software video analyzer Tracker.

\section{Theory}

The theory of light diffraction from a grating is a very well explained phenomenon in every book of general physics $[1,2]$.

Figure 1 shows parallel light rays crossing a diffraction grating and hitting to a target. If $d$ is the spacing between centers of adjacent rulings (grating spacing), $D$ is the distance between the grating and the target, big enough to consider the two rays converging to the target parallels and $x$ is the distance from point $\mathrm{P}$ to the central point $\mathrm{O}$ at the target plane, then the condition of (first) constructive interference at point $\mathrm{P}$ is:

$$
d \sin (\theta)=\lambda
$$

Where $\theta=\tan ^{-1}(x / D)$ and $\lambda$ is the wavelength of light. Therefore, the value $x$ for each wavelength of light is simply given by the relation

$$
\lambda=\frac{d \cdot x}{\sqrt{x^{2}+D^{2}}}
$$

This means that every time a polychromatic light is diffracted, the different colors (different wavelengths) hit the target at different positions $x$ (corresponding to different angles $\theta$ ). The accuracy to distinguish each color depends on resolving power and dispersion of the grating. This means that accuracy is inversely proportional to the grating spacing $d$ and is directly proportional to the number $\mathrm{N}$ of rulings in the diffraction grating. The accuracy of the spectrum lines obtained depends on the slit width used to produce a coherent source of light.

In this work the target will be a photograph camera so the light rays coming from the diffraction grating are focused by a converging lens ensuring that they are parallel when exiting the grating (Fraunhofer diffraction). So the distance $D$ is not important in order to guarantee that rays

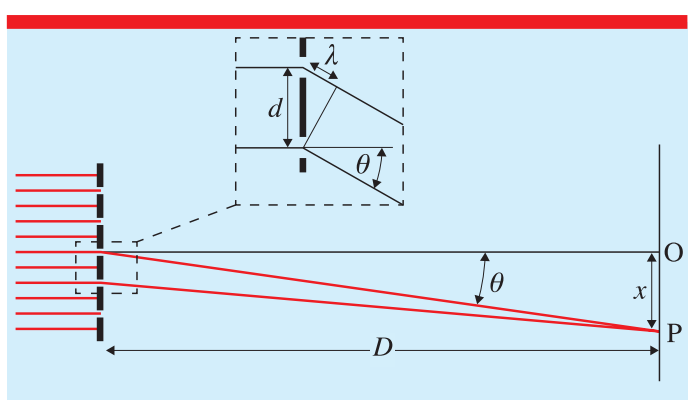

Figure 1. Scheme of the interference of light on a target, after crossing a diffraction grating.

are parallel at point $\mathrm{P}$. Point $\mathrm{O}$ is always the position of slit image (zero-order).

\section{Experimental}

\subsection{About Tracker and wavelength measure-} ment

Tracker is a video analyzer and modelling software created by Douglas Brown [3, 4] and is a project of the Open Source Physics [5]. This software is frequently used for motion studies in mechanics, but also optics as recently reported in [6].

Tracker software has a tool called Line Profile that allows users to measure the bright intensity of the pixels' colors of the image along a straight line, with length defined by the user. One can increase the line width by changing the spread value; image pixels above and below the line are averaged in order to reduce noise and/or increase sensitivity. So we can detect the maximum and minimum intensities of light along a line in an image and measure the distance between them in pixels, or after calibration in meters. This means that we can use this tool to identify the peaks of intensity of each color line in a light spectrum, with more accuracy than the human eye!

Figure 2 shows the Line Profile with spread value of 20 pixels over an interference spectrum of monochromatic light (a He-Ne laser), produced by diffraction grating at a distance of 1.000 $\mathrm{m}$ from the screen, and the graph of the intensity versus position $x$ along the light spectrum. The central peak and four constructive interference maxima for both sides can be easily identified, respectively at $x=0 \mathrm{~cm}, x=2.7 \mathrm{~cm}, x=4.0 \mathrm{~cm}$ and $x=5.4 \mathrm{~cm}$. 
How to build a low cost spectrometer with Tracker for teaching light spectra

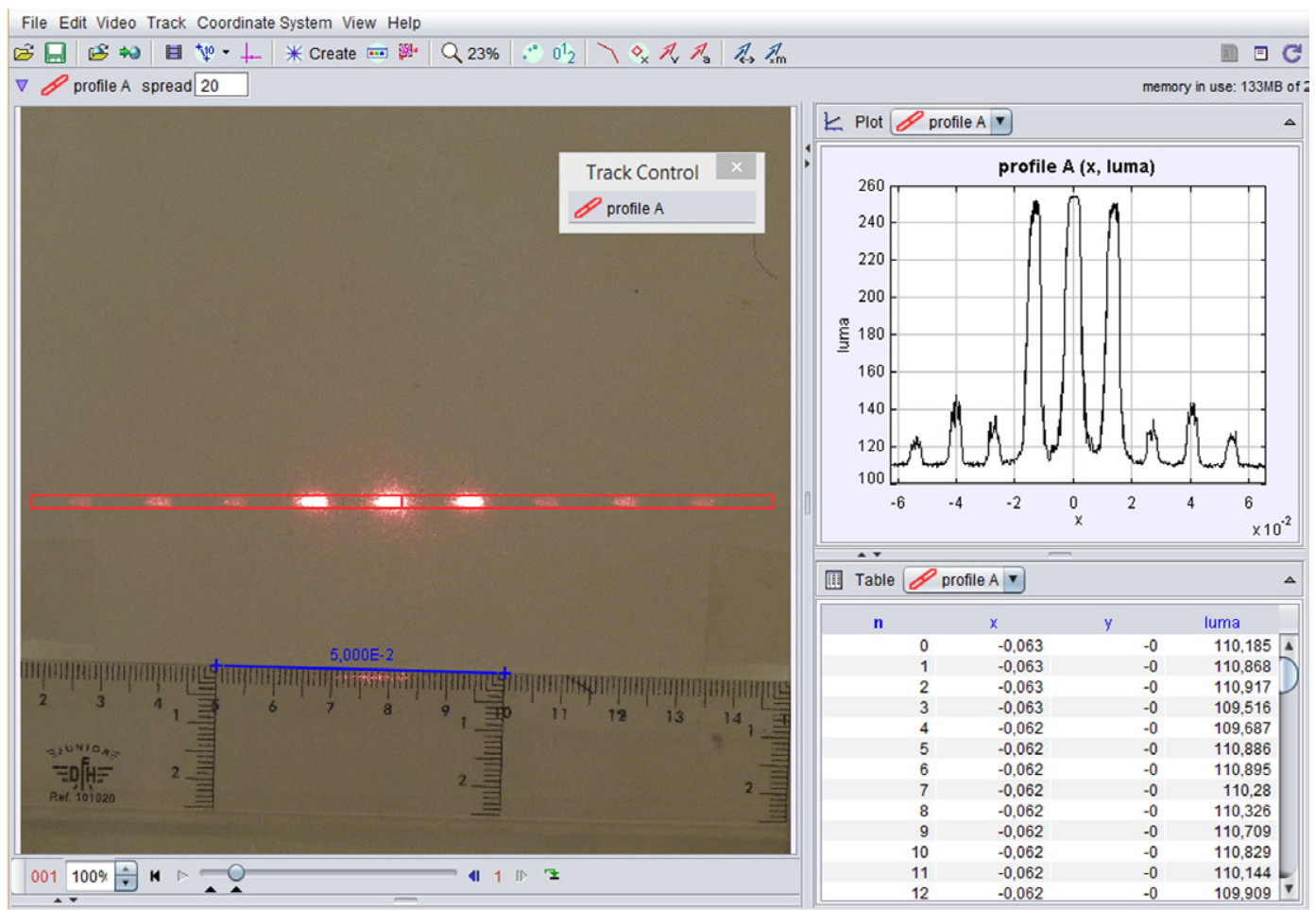

Figure 2. Image obtained from Tracker, for a pattern analysis of a constructive interference. On the left we see the light spots inside a red rectangle (the Line Profile tool) and the calibration ruler in blue. On the top right it is presented the intensity of light as a function of the $x$ position with relation to the central peak [6].

Using equation (2), we obtain the nominal grating spacing $d=46.8 \mu \mathrm{m}$ with an accuracy of $0.3 \mu \mathrm{m}[6]$.

The same measuring technique is here used to find the wavelength $\lambda$ of each color line in the spectra, as we will show in the following.

\subsection{Spectrometer construction}

The spectroscope can be built using a recycled piece of DVD or a commercial transmission diffraction grating (the typical setup for a pocket spectroscope), replacing the eye with the camera (figure 3). There are two ways of obtaining spectral lines. The first and most simple one is placing a grating in front of the camera lens (with some adhesive tape), and then make a thin slit to put it in front of the light source, in order to create a thin line spectrum. In our case we used two razor blades close to each other. This setup usually requires a darkened room.

The other way is creating an opaque tube with a thin slit at one side, and the other side is

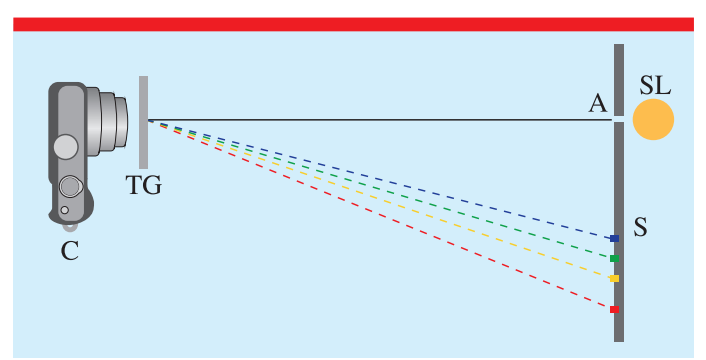

Figure 3. Basic experimental setup. C-digital camera; TG-transmission grating; S-screen; SLspectral lamp; A—aperture.

assembled to the camera lens where the diffraction grating is placed with adhesive tape (figure 4). With this we eliminate the problem of the dispersion of light around the slit, providing beautiful photos of spectra even in the presence of daylight. It has also the advantage to require the calibration procedure only once.

The slit aperture must be narrow enough to resolve the spectral lines detected by the camera. The width of the lines is important in determining 


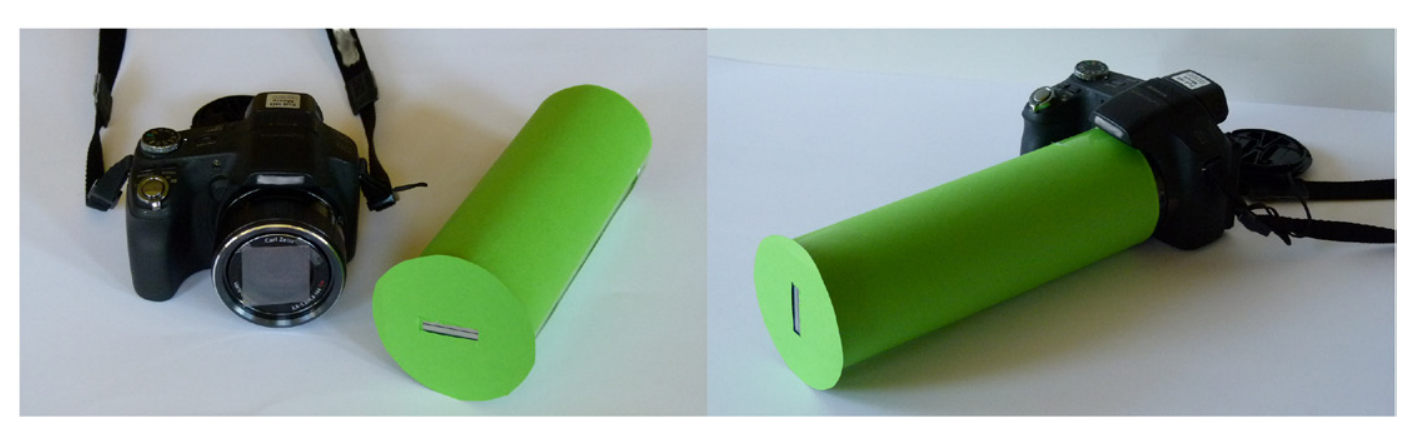

Figure 4. Photos representing the opaque tube with a slit at one end, the camera with the diffraction grating and both parts assembled. The tube guarantees that distance $D$ remains constant for all images as well as the relative position of the slit in the images.

the correct wavelength of each line. The half-with of the each line of the spectrum $\left(\Delta \theta_{\mathrm{hw}}\right)$ at angle $\theta$, is inversely proportional to its number of rulings $(N)$, to the distance $d$ between rulings and to $\cos (\theta)$, and is directly proportional to the wavelength of light [7]:

$$
\Delta \theta_{\mathrm{hw}}=\frac{\lambda}{N d \cos \theta}
$$

This explains why the width $\left(2 \Delta \theta_{\mathrm{hw}}\right)$ increases progressively from blue to red spectral lines for each spectrum order. Therefore, when using a diffraction grating it is better to assure that it covers the entire camera lens, thus increasing the number $N$ of rulings in order to reduce the width of the spectrum lines.

The positions of the colors on the screen, as seen from the camera, correspond to the different wavelengths within the light beam. These wavelengths can be computed using Tracker tools, after calibration of the device (shown in the following). The transmission grating needs to be chosen according to the camera lens: a high number of lines $\mathrm{mm}^{-1}$ increases the spectral resolution, but it also increases the diffracting angle which can be a problem if the spectral lines go out of the optical field of view.

The novelty in our work is that with very simple, common and affordable materials such as a camera and a diffraction grating, we can build a spectrometer with very good resolution (in our case using a 14 Mpixel Panasonic Lumix FZ100 camera and a diffraction grating of 530 lines $\mathrm{mm}^{-1}$ we have a resolution of about $2 \mathrm{~nm} /$ pixel, however the resolution depends on the quality of the image). This can be calibrated using only one well-known light source, and used for qualitative and quantitative teaching purposes, by means of freeware video analyser software.

So far we have considered the ideal situation when all spectral lines are aligned in the plane of the screen. However this may not be entirely true because of the lens distortion.

Most lenses have some distortion, either pincushion or barrel, which usually decreases with the quality of the lens design and the number of elements that compose the lens. This aberration can be approximated by a parabolic change in the scale, positive or negative, as we move from the center to the border. For good photo cameras this effect can be negligible (for first order lines), in the order of other experimental errors but it can be important in cheaper cameras. To correct this effect we can make a quadratic fit centered at the image center or use proper software to correct the image distortion like the Radial Distortion filter available in Tracker or a correction lens tool in other image treatment software. A spectrum image formed near the center of the lens helps to minimize the distortion error. Lines near the border of the images have a significant error due to the distortion lens effect.

\subsection{Spectrometer calibration}

The calibration is done using a light source with a well-known spectrum.

Commercial spectrometers are usually factory calibrated. Spectral calibrating lamps are supplied for checking long term calibration. Error differences can be uploaded to provide correction. Most suppliers sell mercury-based lamps for UV and visible calibration, argon or neon for red 
How to build a low cost spectrometer with Tracker for teaching light spectra
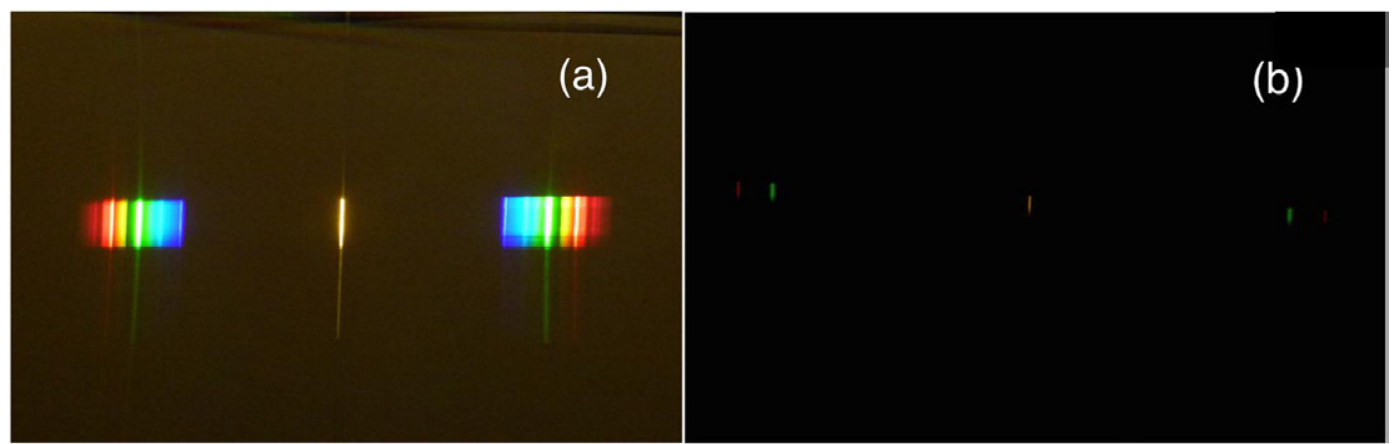

Figure 5. Spectra lines for a compact fluorescent lamp. (a) High light intensity into the camera originates bright spectral lines, but the spectrum is blurred; (b) Low light intensity masks the spectra and only the brightest lines can be observed. In these two photos, only the shutter speed was changed. Calibration must be done with a simple laser light or a reference spectral lamp.

and IR calibration, and a mixture of mercury and argon for the whole spectrum.

The calibration must be done before measuring the spectra of any light source, because it depends on the experimental conditions of the setup-usually a simple shot with a calibrating light source is enough.

In schools, teachers can also use any available spectral lights like sodium or helium lamps, but we do not recommend hydrogen lamps due to the complex spectrum of $\mathrm{H}_{2}$. Teachers can also use other available light sources, such as commercial He-Ne lasers. Usually the manufacturer gives the wavelength information of the ray and the error value. We don't recommend pointing the laser directly into the camera lens because this can damage the camera CCD; instead point it to a white background (e.g. a white sheet of paper) behind the slit.

After capturing an image of the spectrum, Tracker can be used to calibrate the image dimensions as described before. Depending on the accuracy needed for the experiment, it is possible to consider approximately a linear relation between the wavelength and the distance $x$ or we can consider the truly non liner relation between them given by equation (2).

\subsection{Experimental measurements}

The first thing to implement is the experimental setup. We can place the camera with the grating in front of the aperture from which light comes from the spectral lamp, as represented in figure 3. The relative positions of the camera and screen must not move along all the experiment (otherwise, calibration must be restarted). Using the opaque tube setup we avoid this problem. Photo resolution must also be fixed for all the photos.

We need to control the light intensity coming into the camera using the shutter speed: high light intensity creates bright spectral lines but can blur the spectra (figure 5(a)); however with very low light intensity, some lines can become 'masked' in darkness (figure 5(b)). Therefore some photos must be taken in order to know the best experimental shooting conditions and for identifying the spectral lines. The photos can be taken in normal room light or in a dark room for more beautiful spectrum photos. The darker the image background the more visible the intensity peaks of the spectrum lines are. With the tube setup we don't need to look for a dark room.

Starting with the simplest spectra, the Sodium, the yellow spectral line (which, in fact, are two) at $590 \mathrm{~nm}$ corresponds in figure 6 to an average displacement $x$ from the central line of about 330.4 pixels. The average displacement was used to minimize the misalignment between the camera, the grating and the plane of the slit. From equation (2), we can calculate the effective distance $D$ in pixels, which will be fixed for the whole experiment and corresponds to our most important calibration parameter.

The conversion from pixels to wavelength can be done using an Excel sheet, or in a more 


\section{Rodrigues et al}
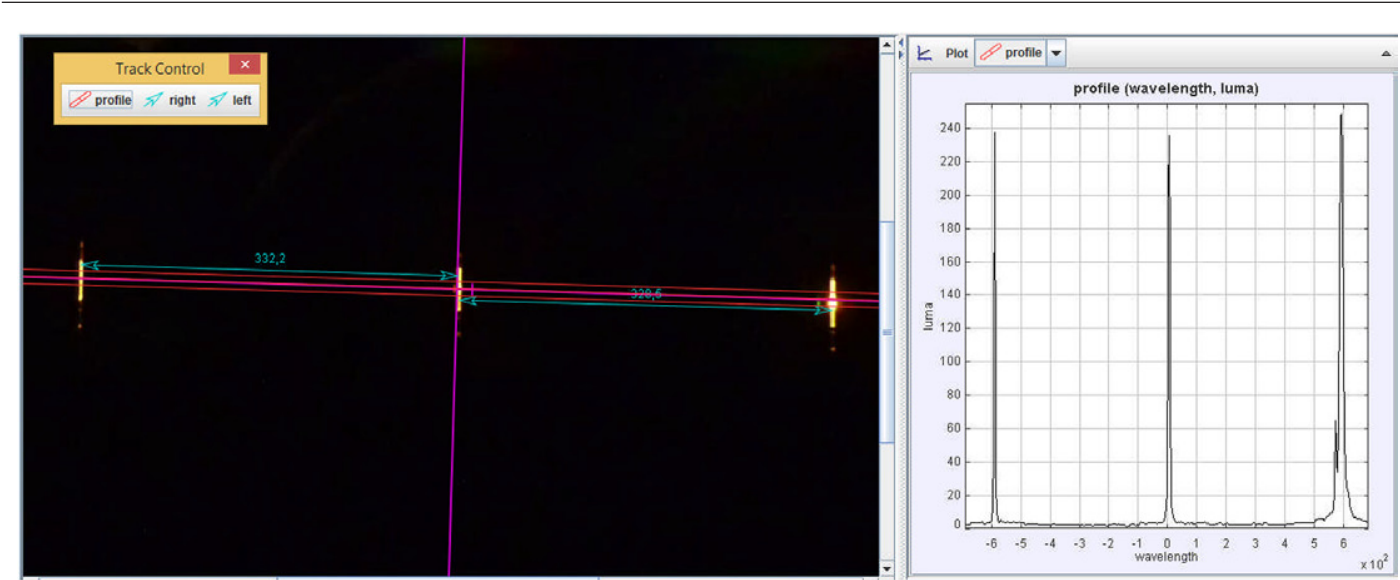

Figure 6. Plot of Tracker main view showing the central and the yellow spectral lines of sodium, and the corresponding light profiles. In the photo (left side), the distance is measured in pixels; in the plot (right side), the distance is converted to wavelength via equation (2). The spectrum was obtained with a grating of 530 lines $\mathrm{mm}^{-1}$.

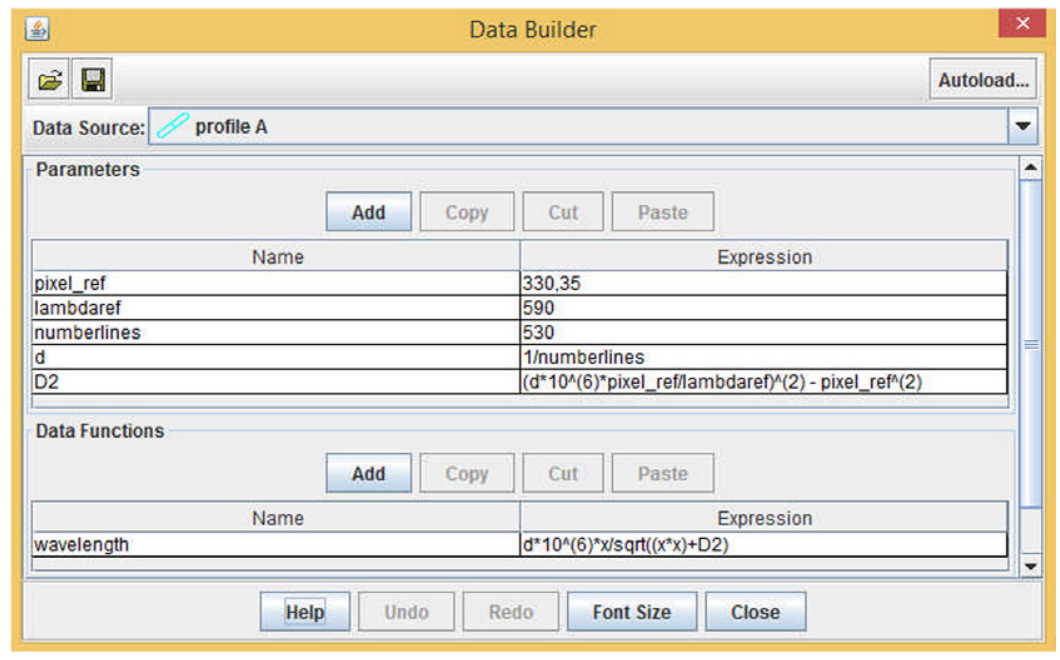

Figure 7. Data Builder window, showing the parameters to be inserted and the Data Function, namely wavelength calculated from equation (2).

easy way with Tracker using equation (2) in the Data Builder tool. As shown in figure 7, the user has only to insert: (1) the average distance (in pixels) between the reference line and the central line in the pixel_ref parameter; (2) the corresponding wavelength of the reference light in the lambdaref parameter, and (3) the number of lines per mm (numberlines parameter) of the diffraction grating used. Tracker automatically calculates the wavelength according to equation (2), for each distance $x$ measured. This procedure allows students to analyze spectrum light directly in the Tracker's plot window, as shown in figure 6.
Depending on the students level of knowledge, the teacher may however decide to implement a more simple and easy way of calibration, considering that the wavelength has approximately a linear relation with the position $x$ in the spectrum.

\section{Results and discussion}

The analysis of the spectra obtained for several light sources is presented in figure 8. Figure 8(a) shows the spectra of a helium lamp and figure $8(\mathrm{~b})$ the spectra of a mercury lamp. These spectra were used to confirm the calibration of the spectrometer. The spectra were obtained with a grating 
How to build a low cost spectrometer with Tracker for teaching light spectra
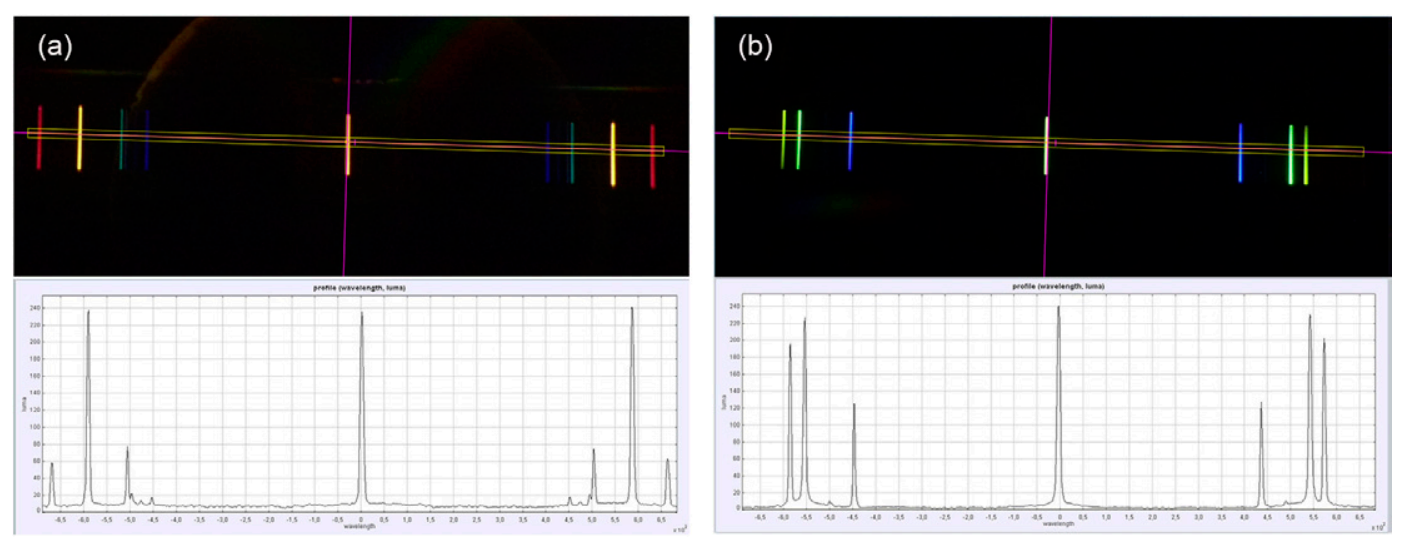

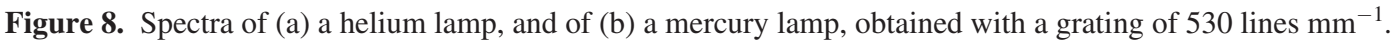

Table 1. Wavelengths of spectral lines of mercury and helium lamps, after calibration with the sodium lamp.

\begin{tabular}{|c|c|c|c|c|c|c|c|}
\hline \multirow[b]{2}{*}{ Colour } & \multicolumn{3}{|c|}{ Mercury } & \multirow[b]{2}{*}{ Colour } & \multicolumn{3}{|c|}{ Helium } \\
\hline & $\begin{array}{l}\text { Experimental } \\
(\mathrm{nm})\end{array}$ & $\begin{array}{l}\text { Literature } \\
(\mathrm{nm})\end{array}$ & $\begin{array}{l}\text { Error } \\
(\%)\end{array}$ & & $\begin{array}{l}\text { Experimental } \\
(\mathrm{nm})\end{array}$ & $\begin{array}{l}\text { Literature } \\
(\mathrm{nm})\end{array}$ & $\begin{array}{l}\text { Error } \\
(\%)\end{array}$ \\
\hline Blue & 441 & 436 & 1.1 & blue & 453 & 447 & 1.3 \\
\hline Ggreen & 548 & 546 & 0.4 & green & 506 & 502 & 0.8 \\
\hline \multirow[t]{2}{*}{ Yellow } & 580 & 579 & 0.2 & yellow & 588 & 588 & 0.0 \\
\hline & & & & red & 664 & 668 & 0.6 \\
\hline
\end{tabular}

Note: The resolution is about $2 \mathrm{~nm}$. The experimental values are compared with those found in literature (http://hyperphysics. phy-astr.gsu.edu/hbase/quantum/atspect.html\#c1 for helium; http://hyperphysics.phy-astr.gsu.edu/hbase/quantum/atspect2. $\mathrm{html} \# \mathrm{c} 2$ for mercury).

of 530 lines $\mathrm{mm}^{-1}$ (distance between rulings, $d=1 / 530 \mathrm{~mm})$.

The maxima light spots for each spectrum were measured with Tracker's measuring tool Line Profile as described in figure 2. The average distances from the central line to each line of the first constructive interference pattern for all lamps, was measured based on the calibration with the sodium light, and the corresponding wavelengths calculated. The values are compared, in table 1 , to the different spectral lines referred in literature, for confirmation.

We can now look to the compact fluorescent lamp spectrum, measure the wavelength of each spectral line and try to identify a particular pattern of lines, corresponding to the presence of a particular substance. Figure 9 shows that the spectrum obtained contains relevant lines at blue (442 nm), light blue $(492 \mathrm{~nm})$, green $(547 \mathrm{~nm})$, yellow $(587 \mathrm{~nm})$ and red $(612 \mathrm{~nm})$. The relevant data are shown in table 2.

Results above confirm that the major mercury lines are easily identified on the compact fluorescent light spectrum, which means that compact fluorescent lamps have mercury in their composition. A simple educational consequence of this result is that commercial compact fluorescent lamps can be used to calibrate the low-cost spectrometer, and therefore schools do not need to acquire helium, mercury, sodium lamps or any other expensive calibration system.

\section{Strategic implementation at school}

Our spectrometer, because of its simplicity, can be used to introduce diffraction phenomena and spectra study. There are many ways to implement this setup: for example, teachers can take some photos of spectra and give them to their students for analyzing; maybe the best way is to make a video with the photos to provide one single file. However we recommend that students, individually or in group, make their own photos after some planning with the teacher's help. This will engage students to this experiment and provide motivation and hopefully a more significant learning. 

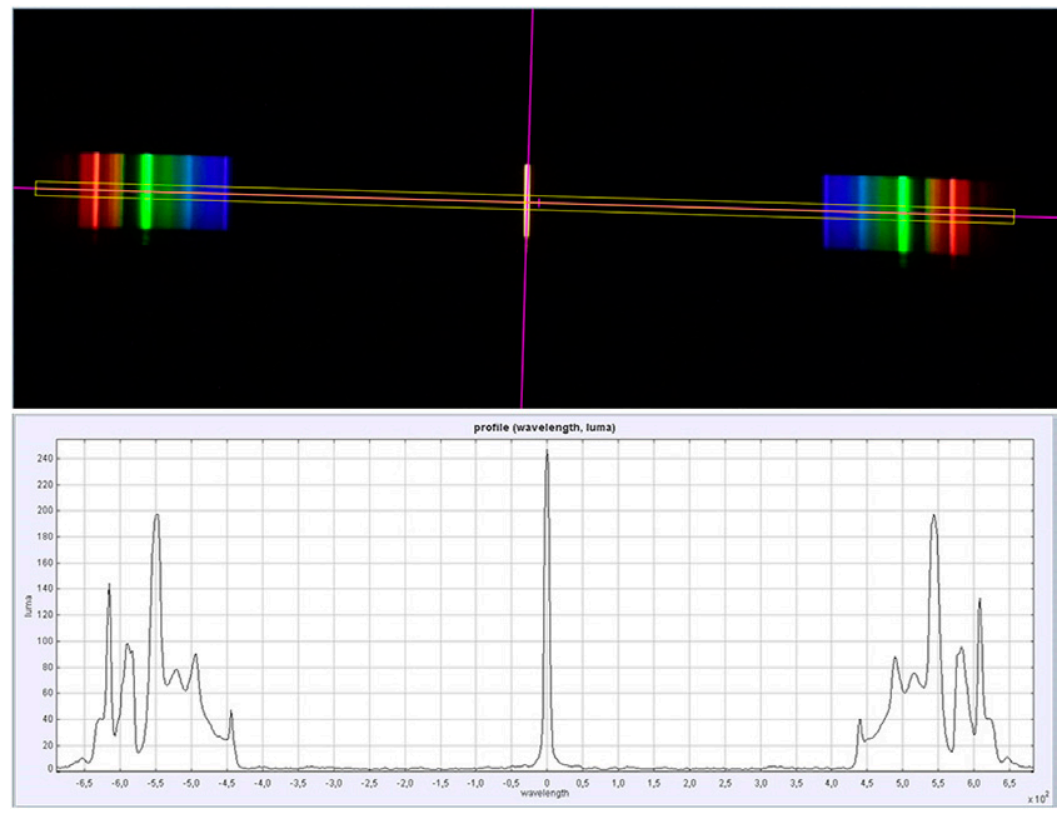

Figure 9. Spectrum of a compact fluorescent lamp, obtained with a grating of 530 lines $\mathrm{mm}^{-1}$.

Table 2. Wavelengths of spectral lines of a compact fluorescent light source, after calibration with the sodium lamp.

\begin{tabular}{|c|c|c|c|}
\hline & Economizer & Mercury & \\
\hline Colour & $\begin{array}{l}\text { Experimental } \\
(\mathrm{nm})\end{array}$ & $\begin{array}{l}\text { Literature } \\
(\mathrm{nm})\end{array}$ & $\begin{array}{l}\text { Error } \\
(\%)\end{array}$ \\
\hline Blue & 442 & 436 & 1.4 \\
\hline Light blue & 492 & & \\
\hline Green & 547 & 546 & 0.2 \\
\hline Yellow & 587 & 579 & 1.4 \\
\hline Red & 612 & & \\
\hline
\end{tabular}

Note: The resolution is about $2 \mathrm{~nm}$. The experimental values are compared with those found in literature for the mercury light.

They can even build their own spectroscope tube, use it with their camera or cellphone, and discuss the relevant parameters for the construction of their spectroscope.

The spectrum analysis can be made with Tracker, in the way shown in this work. Teachers must help students in their task according to their grade level.

There are several activities that can be done with this experiment, for example: (1) the study of emission light spectrum of substances and materials that act like light sources, (2) the reflection spectrum of surfaces, and (3) the light absorption spectrum in solutions. Teachers can implement this experiment as a school project, as homework, or as a laboratory activity. In the classroom, teachers can talk about spectra and/or diffraction phenomena, and simultaneously show how a spectrometer works.

\section{Conclusions}

The main idea for the development of this setup is to provide an easy and low-cost experiment for teaching light spectra in schools and universities. If a school cannot afford a reference spectral lamp such as sodium or mercury, teachers can improvise with a compact fluorescent lamp since the major mercury lines are easily identified on its spectrum. Therefore teachers and students can use compact fluorescent lamps to calibrate the spectrometer.

The advantage of this setup is to provide an instrument that anyone can build and use everywhere, either as a school project, as homework or as laboratory activities. Students can even show to their family the difference between light sources such as LEDs, fluorescent lamps and street lamps. The spectroscope helps to learn better about spectra and/or diffraction phenomena, to investigate the characteristics of light sources (continuous or discontinuous), to study the light spectra of elements (the identity card of elements) and how 
How to build a low cost spectrometer with Tracker for teaching light spectra

scientists know the constitution of matter (for example, chemical solutions or astronomical bodies and stars).

\section{Acknowledgments}

This work is partially financed by the FCTFundação para a Ciência e a Tecnologia (Portuguese Foundation for Science and Technology) within projects UID/NAN/50024/2013 and UID/ EEA/50014/2013.

Received 28 July 2015, in final form 24 August 2015 Accepted for publication 9 September 2015 doi:10.1088/0031-9120/51/1/014002

\section{References}

[1] Serway R A and Beichner R J 2000 Physics for Scientists and Engineers with Modern Physics 5th edn (Philadelphia, PA: Saunders) pp 1224-7

[2] Young H D and Freedman R A 1996 University Physics 9th edn (New York: Addison-Wesley) pp 1176-9

[3] Brown D 2008 Video modeling: combining dynamic model simulations with traditional video analysis American Association of Physics Teachers Summer Meeting (Edmonton) (www.cabrillo.edu/ dbrown/tracker/)

[4] Brown D and Cox A J 2009 Innovative uses of video analysis Phys. Teach. 47 145-50

[5] OSP 2011 Open Source Physics Collection on ComPADRE (www.compadre.org/OSP/)

[6] Rodrigues M and Carvalho P S 2014 Teaching optical phenomena with tracker Phys. Educ. 49 671-7
[7] Resnick R, Halliday D and Walker J 2011

Fundamentals of Physics 9th edn (New York: Wiley) pp 1006-7

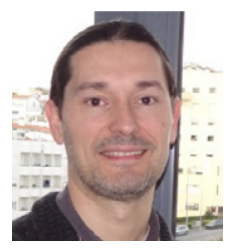

Marcelo José Rodrigues is a middle and high school teacher and researcher at IFMUP. His research interests are interactive educational materials, physics education and teaching training.

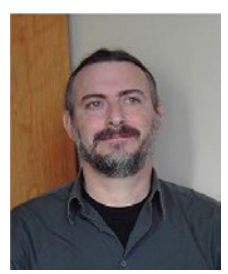

Manuel B Marques received a degree in physics from Porto University in 1983, and a $\mathrm{PhD}$ degree in physics from the same university in 1991. His thesis work was on nonlinear coupling to optical waveguides. From 1993 to 2001, he was a member of Centro de Física do Porto, where he worked on fiber lasers and integrated optics. He joined INESC Porto, Portugal, in 2002, where he has worked on fiber lasers and sensors. He has been an assistant professor in the Physics Department of Porto University since 1991.

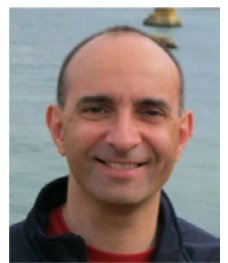

Paulo Simeão Carvalho is an assistan professor at the Department of Physics and Astronomy, Faculty of Sciences, University of Porto since 1998. He is also researcher at IFIMUP and member of UEC (Unit for Teaching of Sciences). His research interests are multimedia curricular materials, physics education and teaching training (initial and continuous). 